\title{
Avaliação da repetibilidade no melhoramento de famílias de maracujazeiro ${ }^{(1)}$
}

\author{
Leonarda Grillo Neves ${ }^{2 *}$, Claudio Horst Bruckner ${ }^{3}$, Cosme Damião Cruz ${ }^{4}$, Marco Antonio Aparecido Barelli ${ }^{2}$
}

\section{RESUMO}

Tem-se procurado definir, em programas de melhoramento, o período mínimo de avaliações para comparação de genótipos, o qual é de longo prazo. Este trabalho teve como objetivo estimar o coeficiente de repetibilidade, por meio de análise de variância e de métodos multivariados, das características físicas e químicas importantes no processo de seleção de frutos do maracujazeiro, para uma predição acurada e eficiente. Houve concordância nas magnitudes dos coeficientes de repetibilidade obtidas pelos diferentes métodos, conferindo-lhes maior confiabilidade. As estimativas do coeficiente de repetibilidade obtidas pelo método da análise de variância foram sempre menores do que as estimativas obtidas pelos demais métodos. Os resultados demonstraram que há confiabilidade de $80 \%$ no processo seletivo a partir de 10 medições nas características avaliadas.

Palavras -chave: Modelo biométrico, Passiflora edulis Sims, seleção.

\section{ABSTRACT}

\section{Repeatability in breeding of passion-fruit families}

Breeding programs seek to determine the minimum period of genotype evaluation, which is a long term process. This study aimed to estimate the repeatability coefficient, using analysis of variance and multivariate methods of physical and chemical characteristics that are important for passion-fruit selection to achieve accurate and efficient predictions. There was agreement on the magnitude of the repeatability coefficients obtained by different methods, increasing their reliability. The estimates of the repeatability coefficient obtained by the analysis of variance were always lower than those obtained by other methods. The results showed that there is $80 \%$ confiability in the selection process from 10 measurements taken on the evaluated traits.

Key words: Biometric model, Passiflora edulis Sims, selection.

\footnotetext{
Recebido para publicação em maio de 2009 e aprovado em agosto de 2010

${ }^{1}$ Parte da tese de doutorado apresentada ao curso de pós-graduação Genética e Melhoramento da Universidade Federal de Viçosa (UFV), para obtenção do grau de Doutora em Genética e Melhoramento

${ }^{2}$ Engenheiros-Agrônomos, Doutores, Departamento de Agronomia, Universidade do Estado do Mato Grosso, Avenida São João, s/nº ,Cavalhada, 78200-000, Cáceres, MT, Brasil. leonardaneves@unemat.br.* Autora para correspondência

${ }^{3}$ Engenheiro-agrônomo, Doutor, Departamento de Fitotecnia, Universidade Federal de Viçosa, Avenida Peter Henry Rolfs, s/n, 36570-000, Viçosa , MG, Brasil. bruckner@ufv.br ${ }^{4}$ Engenheiro-agrônomo, Doutor, Departamento de Biologia Geral, Universidade Federal de Viçosa, Avenida Peter Henry Rolfs, s/n, , 36570-000, Viçosa , MG, Brasil. cdcruz@ufv.br
} 


\section{INTRODUÇÃO}

O maracujazeiro amarelo (Passiflora edulis Sims) tem despertado grande interesse dos fruticultores, face à sua rápida produção em relação a outras fruteiras e à sua grande aceitação no mercado. Seus frutos destinam-se ao mercado in natura e para a industrialização, sendo sua importância econômica representada, principalmente, pelo suco integral e concentrado, com sabor característico (Rocha et al., 2001; Gonçalves, 2007). O Brasil, maior produtor mundial da fruta, tem produção em torno de 615 mil toneladas por ano, com produtividade média, porém, de somente 13,9 t/ha (Agrianual, 2010).

A importância da cultura do maracujazeiro no Brasil vem crescendo. Entretanto, entre os problemas mais significativos no cultivo do maracujazeiro estão a baixa produtividade e a desuniformidade da produção nas diferentes épocas de colheita dos pomares, além da desuniformidade da qualidade dos frutos. É necessário reduzir as diferenças entre as plantas com relação a sua capacidade produtiva e aumentar a qualidade do fruto, objetivado melhorar a padronização, incentivada pelos preços mais atrativos da fruta de qualidade superior. Porém, apesar dessa necessidade, ainda são escassos os programas de melhoramento genético do maracujazeiro no Brasil.

O coeficiente de repetibilidade surge como uma possibilidade de modelo biométrico, por permitir tomar mais de uma medida no mesmo indivíduo, definindo o limite superior da herdabilidade e do grau de determinação. A repetibilidade varia de 0,00 a 1,00 , sendo $r=1,00$ a repetibilidade máxima, verificada quando uma das características manifesta-se com muita constância. Portanto, valores altos da estimativa da repetibilidade de uma dada característica indicam que é possível predizer o valor genético real do indivíduo, com um número relativamente pequeno de avaliações. Além disso, a repetibilidade representa o limite superior da herdabilidade $\left(\mathrm{h}^{2}\right)$, sendo que é bem mais fácil de ser estimada, pois $\mathrm{h}^{2}$ exige cruzamentos controlados e estudo de progênies (Falconer, 1981). A repetibilidade expressa a proporção da variância total que é explicada pelas variações proporcionadas pelo genótipo. A repetibilidade provavelmente irá variar com a natureza de cada um dos caracteres que serão avaliados (Cruz et al., 2004).

No processo de seleção de genótipos superiores em espécies frutíferas, é necessária a realização de avaliações periódicas que, com frequência, envolvem grande número de experimentos, vários ciclos de seleção e várias avaliações em diferentes caracteres, significando o emprego considerável de tempo e custo. Em vista disso, ao se escolher um genótipo superior de maracujazeiro espera-se que sua superioridade perdure. A veracidade desse bom desempenho poderá ser comprovada pelo coeficien- te de repetibilidade das características estudadas nos programas de melhoramento. Com esse coeficiente é possível estimar o quanto as medições de um dado caráter apresentam respostas que se repetem, em um mesmo genótipo (Cruz et al., 2004). O método mais empregado para estimar o coeficiente de repetibilidade tem-se baseado nos componentes da análise de variância. No entanto, métodos multivariados, utilizando componentes principais, têm sido propostos por Abeywardena (1972) e Rutledge (1974), que consideram algumas situações em relação à periodicidade dos genótipos em relação à manifestação de determinados caracteres.

Este trabalho teve como objetivo estimar o coeficiente de repetibilidade, por meio de análise de variância e de métodos multivariados, para uma predição acurada e eficiente no melhoramento de famílias de maracujazeiro.

\section{MATERIAL E MÉTODOS}

Foram avaliadas, no ano de 2006, progênies descendentes de plantas da área da Empresa Bela Joana, em Campos dos Goytacazes-RJ, sendo essas plantas oriundas de populações de São Francisco do Itabapoana, Maguary e Yellow Máster. Os cruzamentos que originaram as progênies foram realizados em Campos dos Goytacazes e seguiram o procedimento descrito por Hallauer e Miranda Filho (1988), com base no Delineamento I de Comstock e Robinson, num arranjo em SETS (grupo de tratamentos), composto de 03 Sets. Para a realização deste trabalho, as 113 famílias (irmãos-completos e meios-irmãos) foram avaliadas em Viçosa, Minas Gerais.

Os frutos do maracujazeiro foram levados para análises no Laboratório de Análises de Frutas, do Departamento de Fitotecnia, da Universidade Federal de Viçosa. No Laboratório, o trabalho foi desenvolvido em delineamento inteiramente casualizado, e consistiu na avaliação das 113 famílias, nas quais as medições fenotípicas foram repetidas por dez vezes.

Os caracteres quantificados para o estudo de repetibilidade foram: peso de fruto (PF), medido com balança digital e expresso em gramas; peso de polpa com semente (PP), medido com balança digital e expresso em gramas; espessura da casca (EC), medido com paquímetro digital e expresso em milímetros; diâmetro equatorial e comprimento de fruto (LF e CF, respectivamente), medidos com paquímetro e expresso em milímetros e teor de sólido solúveis totais ( ${ }^{\mathrm{B}} \mathrm{Brix}$ ) (SST), determinados por refratometria.

As estimativas dos coeficientes de repetibilidade foram obtidas pelos métodos de análise de variância (ANOVA); componentes principais (CP), com base na matriz de correlações e de covariâncias; e análise estrutural (AE), com base na matriz de correlações. 
No método de análise de variância, o coeficiente de repetibilidade foi estimado por meio dos resultados da análise de variância, segundo o modelo descrito por Cruz et al, (2004):

$\mathrm{Y}_{\mathrm{ij}}: \mu+\mathrm{g}_{\mathrm{i}}+\varepsilon_{\mathrm{ij}}$, em que:

$\mathrm{Y}_{\mathrm{ij}}$ : observação referente ao i-ésimo ambiente (época de avaliação);

$\mu$ : média geral;

$\mathrm{g}_{\mathrm{i}}$ : efeito aleatório da i-ésima família sob a influência do ambiente permanente $(\mathrm{i}=1,2, \ldots, \mathrm{p})$;

$\varepsilon_{\mathrm{ij}}$ : efeito do ambiente temporário associado à j-ésima medição na i-ésima família $(j=1,2, \ldots . \eta)$.

O coeficiente de repetibilidade foi dado por:

$$
r=\hat{\rho}=\frac{\operatorname{Cov}\left(Y_{i j}, Y_{i j}\right)}{\sqrt{\hat{V}\left(Y_{i j}\right) \hat{V}\left(Y_{i j}{ }^{\prime}\right)}}=\frac{\hat{\sigma}_{g}^{2}}{\hat{\sigma}_{Y}^{2}}=\frac{\hat{\sigma}_{g}^{2}}{\hat{\sigma}^{2}+\hat{\sigma}_{g}^{2}} \text {, em que: }
$$

$\mathrm{Y}_{\mathrm{ij}}$ e $\mathrm{Y}_{\mathrm{ij}}{ }^{\prime}$ são as diferentes medidas, realizadas num mesmo indivíduo.

Foram estimados os resultados da análise de variância para cada caráter descrito e estimados os componentes de variância associados aos efeitos genético e residual. As estimativas dos coeficientes de repetibilidade foram obtidas pelo método dos componentes principais, com base tanto na matriz de correlações, como na matriz de covariâncias entre cada par de medições, avaliadas nas diferentes famílias de maracujazeiro amarelo.

Também foram obtidas as estimativas de repetibilidade pelo método da análise estrutural, com base na matriz de correlações entre as famílias em cada par de avaliação, conforme metodologia proposta por Mansour et al. (1981). De acordo com Cruz et al. (2004), este estimador é a média aritmética das correlações fenotípicas entre genótipos, considerando cada par de medições.

Estimado o coeficiente de repetibilidade (r), a estimativa do número de medições $\left(\mathrm{n}_{0}\right)$ necessárias para se predizer o valor real dos indivíduos com o valor de determinação genotípica $\left(\mathrm{R}^{2}\right)$ desejado foi obtida pela expressão, seguindo a metodologia de Cruz et al. (2004):

$\eta_{\mathrm{o}}=\frac{\mathrm{R}^{2}(1-\hat{\mathrm{r}})}{\left(1-\mathrm{R}^{2}\right) \hat{\mathrm{r}}}$

Segundo o método descrito por Cruz et al. (2004), o coeficiente de determinação genotípica $\left(\mathrm{R}^{2}\right)$, que representou a porcentagem de certeza da predição do valor real dos indivíduos (maracujazeiros) selecionados com base em $n$ medições foi obtido pela equação:

$$
\mathrm{R}^{2}=\frac{\eta \mathrm{r}}{1+\mathrm{r}(\eta-1)}
$$

Rev. Ceres, Viçosa, v. 57, n.4, p. 480-485, jul/ago, 2010
Todas as estimativas foram obtidas por meio do procedimento repetibilidade do programa computacional estatístico GENES (Cruz, 2006).

\section{RESULTADOS E DISCUSSÃO}

Houve diferença, a $1 \%$ de probabilidade, entre as famílias de irmãos completos e meios-irmãos, indicando a existência de variabilidade genética entre as famílias de maracujazeiros estudadas e, assim, perspectivas positivas na obtenção de ganhos genéticos por meio de seleção (Tabela 1). Esse fato indica a existência de heterogeneidade entre as famílias, com possibilidade de auxiliar na identificação de famílias de irmãos completos e meiosirmãos, que deverão ser incluídas no próximo ciclo de cruzamentos.

Em média, as famílias de maracujazeiro amarelo do presente estudo apresentaram características morfoagronômicas desejáveis, ou seja, frutos com pesos médios de 200 gramas, que estão acima dos padrões, levemente ovalados e com Brix em torno de $13^{\circ}$. Entre as características de produtividade pode-se destacar o peso da polpa, que exibiu média de 90,14 gramas, que equivale a $45,04 \%$ de rendimento em suco (Tabela 1). Os frutos de maracujá, atualmente disponíveis no mercado, são bastante variados e têm apresentado as seguintes características: 50130 gramas de peso ; máximo de 36\% de rendimento em suco, e teor de sólidos solúveis totais entre 13 e $18{ }^{\circ} \mathrm{Brix}$ (Ruggiero, 1996).

Os coeficientes de variação obtidos para esses caracteres estiveram dentro dos limites de experimentos avaliados, evidenciando boa precisão experimental. Os caracteres que exibiram os maiores valores foram peso da polpa (PP) e espessura da casca (EC), com 22,83 e 21,17\%, respectivamente, sendo, possivelmente, os mais influenciados pelo ambiente. Por outro lado, o comprimento (CF) e diâmetro equatorial dos frutos (LF) foram os caracteres morfológicos com menores coeficientes de variação (Tabela 1 ).

Tem-se procurado definir o período mínimo de avaliações para comparação de genótipos em programas de melhoramento, o qual é de longo prazo. Nesses programas, não pode haver perda de tempo, avaliando-se os genótipos além do necessário, assim como não se pode avaliar por um período muito pequeno, que pode levar a erros na identificação dos genótipos superiores. As estimativas do coeficiente de repetibilidade obtidas, com relação às seis características avaliadas por meio dos quatro métodos estatísticos utilizados, encontram-se na Tabela 2. Houve concordância nas magnitudes dos coeficientes de repetibilidade, obtidas pelos diferentes métodos, conferindo-lhes maior confiabilidade.

As estimativas do coeficiente de repetibilidade obtidas pelo método da análise de variância foram sempre 
Tabela 1- Resumo da análise de variância dos caracteres peso de fruto (PF), peso de polpa com semente (PP), espessura da casca (EC), diâmetro equatorial e comprimento de fruto (LF) (CF) e teor de sólidos solúveis totais ( ${ }^{\circ}$ Brix) (SST), em 10 medições, em 113 famílias de maracujazeiro amarelo (Passiflora edulis Sims), estimativa dos componentes de variância entre famílias e variância ambiental e coeficiente de variação (CV)

\begin{tabular}{|c|c|c|c|c|c|c|c|}
\hline \multirow{2}{*}{$\begin{array}{l}\text { Fonte de } \\
\text { Variação }\end{array}$} & \multirow{2}{*}{$\begin{array}{c}\text { Graus de } \\
\text { Liberdade }\end{array}$} & \multicolumn{6}{|c|}{ Q.M. } \\
\hline & & PF & PP & EC & $\mathbf{L F}$ & $\mathbf{C F}$ & SST \\
\hline Medições & 9 & 29094,27 & 9691,71 & 48,93 & 323,22 & 353,85 & 2,15 \\
\hline Famílias & 112 & $6785,20 * *$ & $2092,19 * *$ & $14,03^{* *}$ & $146,49 * *$ & $344,20 * *$ & $5,14^{* *}$ \\
\hline Resíduo & 1008 & 1035,69 & 340,77 & 2,08 & 15,60 & 26,88 & 0,75 \\
\hline Média & & 200,12 & 90,14 & 7,45 & 76,73 & 86,34 & 13,64 \\
\hline CV\% & & 17,90 & 22,83 & 21,17 & 5,57 & 6,32 & 6,42 \\
\hline
\end{tabular}

** Significativo a $1 \%$ de probabilidade, pelo teste $\mathrm{F}$.

menores do que as estimativas obtidas pelos demais métodos (Tabela 2). Lopes et al (2005), em estudo sobre a repetibilidade em caracteres de frutos de aceroleira, afirmam que as estimativas do coeficiente de repetibilidade, obtidas pelo método da análise de variância, são sempre menores ou iguais às estimativas obtidas pelos demais métodos, e que, pelo método dos componentes principais (baseado na matriz de covariâncias), essas estimativas foram sempre maiores ou iguais às obtidas pelos demais métodos.

No entanto, observando a Tabela 2, as diferenças observadas entre as estimativas obtidas pelos diferentes métodos são pequenas e, na maioria dos casos, não alteram o número de medições em um mesmo nível de precisão. Abeywardena (1971) afirma que o coeficiente de repetibilidade pode ser mais eficientemente estimado por meio da técnica de componentes principais. Numa situação em que o fator periodicidade ocorre, este não pode ser isolado na análise de variância. Com isto, este componente é incluído no erro experimental, elevando seu valor e levando a estimativas não verdadeiras deste componente e, nesta situação, a repetibilidade é subestimada (Abeywardena, 1972; Kendall, 1975; Vasconcellos et al., 1985).

Os coeficientes de repetibilidade dos caracteres de frutos estudados mostraram índices que variaram de $\mathrm{r}$ $=0,30$ a $r=0,54$. Os maiores valores foram registrados para diâmetro equatorial e comprimento de frutos, $\mathrm{r}=$ 0,46 e $r=0,55$, respectivamente, obtidos pelo método de componentes principais, baseado na matriz de correlação e covariância (Tabela 2). Mansour et al. (1981) relatam que um caráter de considerável repetibilidade possui melhor desempenho que os demais, principalmente se apresentar alta associação com a variável desejável. Desta forma, pode-se supor que, pelo fato de os valores de r estimados serem relativamente baixos, seja recomendável a avaliação dos caracteres dos frutos das famílias de maracujazeiro com base em um número relativamente alto de medições, para que, desta forma, haja a possibilidade de proceder à seleção fenotípica simples com objetivo de aumento de produção. Por meio do método dos componentes principais, baseado na matriz de covariância e correlação, as estimativas foram sempre maiores ou iguais às obtidos pelos demais métodos (Tabelas 2).

Os caracteres morfológicos e de produção variaram de 79,75 a 92,26\%, para PP (pelo método de ANOVA) e CF (Componentes Principais), respectivamente. Tal fato pode estar revelando maior precisão para expressar o real valor das plantas para o número de medições efetuadas. Esses resultados demonstram que há alta confiabilidade nos números de medições necessárias para todos os caracteres em estudo. De acordo com Padilha et al. (2003), em estudo para fins de obtenção da estimativa da repetibilidade em caracteres morfológicos e de produção de palmito em pupunheira (Bactris gasipaes Kunth), valor acima de 50\% indica que existe confiabilidade significativa para os números de medições necessárias para os diferentes coeficientes de determinação.

No que diz respeito ao número de medições realizadas para efetuar uma seleção efetiva, com $90 \%$ de precisão $(\eta)$, foi verificado que apenas o comprimento do fruto apresentou um número de medições possível de serem realizadas, ou seja, sete medições por família, resultado obtido pelo método de componentes principais, baseado na matriz de covariância (Tabela 2). Demais caracteres morfológicos exibiram números de medições elevados para uma seleção efetiva, para todos os quatro métodos estatísticos estudados, necessitando de mais de 11 medições por famílias, para 90\% de certeza.

Cardoso (2006) efetuou 32 colheitas em híbridos de pepino (Cucumis sativus L.) e estimou os coeficientes de repetibilidade, com base na análise de componentes principais, relativamente ao caráter produção de frutos por planta, em massa e número, total e comercial. Os resultados permitiram concluir que menos de nove colheitas foram suficientes para analisar as diferenças de produtividade entre os diferentes híbridos, com 95\% de certeza de serem eleitos os mais produtivos. 
Oliveira e Fernandes (2001) estimaram o coeficiente de repetibilidade em caracteres do cacho de açaizeiro (Euterpe oleracea Mart.). A análise da repetibilidade, do número de medições necessárias e do coeficiente de determinação para cada caráter foi obtida pelo método da análise de variância. O maior coeficiente de repetibilidade foi registrado para peso médio de frutos (PMF), variável essa que teve também o maior coeficiente de determinação. Entretanto, o número desejável de repetições para esse caráter deve ser quase o triplo do proposto inicialmente pelos autores. Pelo fato de o coeficiente de repetibilidade expressar o valor máximo de herdabilidade, os autores concluíram que o PMF deve ser usado como parâmetro de seleção em métodos de melhoramento menos rigorosos.

Segundo Faria Neto et al. (2003), pode ser considerado como satisfatório um nível de 90\% de predição na tomada de decisão sobre a superioridade relativa das famílias e, assim, espera-se que, com um maior número de repetições, possa ser alcançada maior precisão na determinação do valor real do coeficiente. Entretanto, o processo seletivo seria impraticável para a avaliação dos caracteres PF, PP, EC e SST, pois seria necessário realizar pelo menos 15, 17, 13 e 15 avaliações, respectivamente, tomando como

Tabela 2 - Estimativa dos coeficientes de repetibilidade ( $\mathrm{r}$ ), coeficientes de determinação $\left(\mathrm{R}^{2}\right)$ e do número de medições calculado $\left(\mathrm{N}_{0}\right)$ para os caracteres peso de fruto (PF), peso de polpa com semente (PP), espessura da casca (EC), diâmetro equatorial e comprimento de fruto (LF) (CF), e teor de sólido solúveis totais ( ${ }^{\circ}$ Brix) (SST), em 10 medições, em 113 famílias de maracujazeiro amarelo (Passiflora edulis Sims)

\begin{tabular}{|c|c|c|c|c|c|c|c|c|c|c|c|c|c|}
\hline \multirow[t]{2}{*}{ Caracter } & \multicolumn{8}{|c|}{ Valor obtido a partir de 10 medições } & \multicolumn{5}{|c|}{$\begin{array}{l}\text { Número de medições necessárias para diferentes } \\
\text { coeficientes de determinação }\end{array}$} \\
\hline & $\widehat{\mathrm{r}}_{1}$ & $\widehat{\mathrm{r}}_{2}$ & $\widehat{\mathrm{r}}_{3}$ & $\hat{\mathrm{r}}_{4}$ & $\begin{array}{c}\mathrm{R}^{2} \\
\%\end{array}$ & $\begin{array}{l}\mathrm{R}^{2} \\
\%\end{array}$ & $\begin{array}{c}\mathrm{R}^{2}{ }_{3} \\
\%\end{array}$ & $\begin{array}{c}\mathrm{R}_{4}^{2} \\
\%\end{array}$ & $\mathrm{R}^{2}$ & $\eta_{01}{ }^{(*)}$ & $\eta_{02}^{(*)}$ & $\eta_{03}{ }^{(*)}$ & $\eta_{04}{ }^{(*)}$ \\
\hline \multirow[t]{5}{*}{$\mathrm{PF}$} & 0,30 & 0,36 & 0,38 & 0,35 & 81,1 & 85,2 & 86,1 & 84,5 & 0,80 & $9(9,3)$ & $7(6,9)$ & $6(6,4)$ & $7(7,3)$ \\
\hline & & & & & & & & & 0,85 & $13(13,2)$ & $10(9,8)$ & $9(9,2)$ & $10(10,4)$ \\
\hline & & & & & & & & & 0,90 & $21(21,0)$ & $16(15,6)$ & $15(14,6)$ & $16(16,4)$ \\
\hline & & & & & & & & & 0,95 & $44(44,3)$ & $33(32,9)$ & $31(30,8)$ & $35(34,8)$ \\
\hline & & & & & & & & & 0,99 & $231(231,1)$ & $172(171,9)$ & $160(160,3)$ & $181(181,4)$ \\
\hline \multirow[t]{5}{*}{$\mathrm{PP}$} & 0,28 & 0,35 & 0,35 & 0,34 & 79,7 & 84,2 & 84,3 & 83,8 & 0,80 & $10(10,1)$ & $7(7,4)$ & $7(7,4)$ & $8(7,7)$ \\
\hline & & & & & & & & & 0,85 & $14(14,4)$ & $11(10,6)$ & $11(10,6)$ & $11(10,9)$ \\
\hline & & & & & & & & & 0,90 & $23(22,8)$ & $17(16,9)$ & $17(16,9)$ & $17(17,4)$ \\
\hline & & & & & & & & & 0,95 & $48(48,2)$ & $36(35,7)$ & $35(35,3)$ & $37(36,7)$ \\
\hline & & & & & & & & & 0,99 & $251(251,3)$ & $186(185,8)$ & $186(185,8)$ & 191(191,3) \\
\hline \multirow[t]{5}{*}{ EC } & 0,32 & 0,44 & 0,40 & 0,43 & 82,2 & 88,8 & 87,1 & 88,2 & 0,80 & $9(8,6)$ & $5(5,0)$ & $6(5,9)$ & $5(5,3)$ \\
\hline & & & & & & & & & 0,85 & $12(12,2)$ & $7(7,1)$ & $8(8,4)$ & $8(7,6)$ \\
\hline & & & & & & & & & 0,90 & $19(19,5)$ & $11(11,3)$ & $13(13,4)$ & $12(11,9)$ \\
\hline & & & & & & & & & 0,95 & $41(41,1)$ & $24(23,8)$ & $28(28,2)$ & $25(25,3)$ \\
\hline & & & & & & & & & 0,99 & $214(214,1)$ & $124(124,0)$ & $147(147,1)$ & $132(131,8)$ \\
\hline \multirow[t]{5}{*}{ LF } & 0,41 & 0,46 & 0,46 & 0,46 & 87,5 & 89,4 & 89,5 & 89,3 & 0,80 & $6(5,7)$ & $5(4,7)$ & $5(4,7)$ & $5(4,8)$ \\
\hline & & & & & & & & & 0,85 & $8(8,1)$ & $7(6,7)$ & $6(6,1)$ & $7(6,7)$ \\
\hline & & & & & & & & & 0,90 & $13(12,8)$ & $11(10,6)$ & $10(10,4)$ & $11(10,7)$ \\
\hline & & & & & & & & & 0,95 & $27(27,2)$ & $22(22,3)$ & $22(22,2)$ & $22(22,4)$ \\
\hline & & & & & & & & & 0,99 & $142(141,6)$ & $116(116,4)$ & $115(115,4)$ & $118(118,0)$ \\
\hline \multirow[t]{5}{*}{$\mathrm{CF}$} & 0,52 & 0,54 & 0,55 & 0,54 & 91,3 & 92,3 & 92,4 & 92,2 & 0,80 & $4(3,8)$ & $3(3,3)$ & $3(3,3)$ & $3(3,4)$ \\
\hline & & & & & & & & & 0,85 & $5(5,4)$ & $5(4,7)$ & $5(4,7)$ & $5(4,8)$ \\
\hline & & & & & & & & & 0,90 & $9(8,6)$ & $8(7,6)$ & $7(7,4)$ & $8(7,6)$ \\
\hline & & & & & & & & & 0,95 & $18(17,9)$ & $16(15,9)$ & $16(15,7)$ & $16(16,1)$ \\
\hline & & & & & & & & & 0,99 & $94(93,8)$ & $83(83,0)$ & $82(81,8)$ & $84(84,1)$ \\
\hline \multirow[t]{5}{*}{ SST } & 0,36 & 0,37 & 0,37 & 0,37 & 85,1 & 85,6 & 85,6 & 85,5 & 0,80 & $7(7,0)$ & $7(6,7)$ & $7(6,7)$ & $7(6,8)$ \\
\hline & & & & & & & & & 0,85 & $10(9,9)$ & $10(9,6)$ & $9(9,6)$ & $10(9,6)$ \\
\hline & & & & & & & & & 0,90 & $16(15,8)$ & $15(15,2)$ & $15(15,1)$ & $15(15,3)$ \\
\hline & & & & & & & & & 0,95 & $33(33,3)$ & $32(32,0)$ & $32(31,8)$ & $32(32,2)$ \\
\hline & & & & & & & & & 0,99 & $173(173,4)$ & $167(166,9)$ & $165(165,8)$ & $168(168,1)$ \\
\hline
\end{tabular}

(*) Número aproximado (número calculado).

Rev. Ceres, Viçosa, v. 57, n.4, p. 480-485, jul/ago, 2010 
base o método dos componentes principais, baseado na matriz de covariância (Tabela 2).

As quantidades necessárias de medições, estimadas neste estudo, tornam-se extremamente trabalhosas e até mesmo inviáveis de serem realizadas em maracujazeiro, pois há a necessidade de grande dispêndio de tempo e mão de obra. Nota-se que é possível obter maiores níveis de precisão, com relação aos caracteres analisados, mediante maior número de medições, nas quais se espera alcançar pelo menos $90 \%$ de determinação do valor real das famílias de maracujazeiro.

\section{CONCLUSÕES}

Houve concordância nas magnitudes dos coeficientes de repetibilidade obtidas pelos diferentes métodos, conferindo-lhes maior confiabilidade.

A confiabilidade do processo seletivo a partir de 10 medições está em torno de $80 \%$ para os caracteres avaliados.

\section{AGRADECIMENTOS}

À Coordenação de Aperfeiçoamento de Pessoal de Nível Superior-CAPES e ao Conselho Nacional de Desenvolvimento Científico e Tecnológico-CNPq, pela concessão de bolsa de Doutorado à primeira autora, assim como auxílio financeiro à pesquisa. Ao professor Alexandre Pio Viana, da UENF, pela cessão das progênies avaliadas neste estudo.

\section{REFERENCIAS}

Agrianual, (2010). Anuário estatístico da agricultura brasileira. São Paulo, FNP, 520p.

Abeywardena V (1972). An application of principal components analysis in genetics. Journal of Genetic, 16:27-51.

Bruckner CH, Meletti LMM, Otoni WC \& Zerbini Junior FM (2002) Maracujazeiro. In: Bruckner CH (Ed.) Melhoramento de fruteiras tropicais. Viçosa, Editora UFV, p. 373-410.

Cardoso AIIC. (2006) Número Mínimo de Colheitas em Pepino Híbrido Estimado Por Meio do Coeficiente de Repetibilidade. Bragantia, 65:591-595.

Cavalcanti JJV, Paiva JR, Barros LM, Crisóstomo JR \&, Corrêa, MPF (2000) Repetibilidade de caracteres de produção e porte da planta em clones de cajueiro-anão precoce. Pesquisa Agropecuária Brasileira, 35:1-6.

Cruz CD (2006) Programa Genes: biometria. Versão Windows; aplicativo computacional em genética e estatística. Viçosa: UFV, 382p.

Cruz CD, Regazzi AJ \& Carneiro PCS. (2004) Modelos Biométricos Aplicados ao Melhoramento Genético. Viçosa, UFV, 480p..

Falconer DS (1981) Introdução à genética quantitativa. Tradução de M.A. Silva e J.C. Silva. Viçosa, UFV, 279p.

Farias Neto JT, Muller AA, Oliveira MSP, Espirito Santo DE \& Silva MA (2003) Variabilidade genética entre duas procedências de açaizeiro (Euterpe oleracea Martus). Boletim de Pesquisa Florestal, 46:97-104.
Kendall MG (1975). Multivariate analysis. New York, MacMillan, $210 \mathrm{p}$.

Lopes R, Bruckner CH, Cruz CD, Lopes MTG \& Freitas GB (2005) Repetibilidade de características do fruto de aceroleira. Pesquisa Agropecuária Brasileira, 36:1-13.

Mansour H, Nordheim EV \& Ruledge JJ (1981) Estimators of repeatability. Theoretical and Applied Genetics, 60:151-156.

Neto JTF, Lins PMP \& Muller AA (2003) Estimativa dos coeficientes de repetibilidade para produção de fruto e albúmen sólido em coqueiro híbrido. Pesquisa Agropecuária Brasileira, 38:12371241.

Oliveira JC (1980) Melhoramento genético de Passiflora edulis f. flavicarpa Deg visando aumento de produtividade. Tese de Livre-Docência. FCAV-UNESP Jaboticabal, 133p.

Oliveira MSP \& Fernandes GLC (2001) Repetibilidade de Caracteres do Cacho de Açaizeiro nas Condições de Belém-PA. Revista Brasileira de Fruticultura, 23:613-616.

Padilha NCC, Oliveira M SP \& Mota, MGC (2003) Estimativa da repetibilidade em caracteres morfológicos e de produção de palmito em pupunheira (Bactris gasipaes Kunth). Revista Árvore, 27:435-442.

Rocha MC, Bonelli ALS, Almeida A \& Collad FH (2001) Efeito do uso de biofertilizante AGROBIO sobre as características físicoquímicas na pós-colheita do maracujá amarelo (Passiflora edulis f. flavicarpa Deg.) no município de Taubaté. Revista Biociências, 7:7-13.

Ruggiero C, São José AR, Volpe CA, Oliveira JC, Durigan JF. Baumgartner JG, Silva J R, Nakamura K. Ferreira ME, Kavati R,\& Pereira VP (1996) Maracujá para exportação: aspectos técnicos da produção. Brasília, MAARA, Secretaria de Desenvolvimento Rural, EMBRAPA-SPI, 64p. (Série Publicações Técnicas FRUPEX, 19).

Rutledge JJ (1974) A scaling which remove bias of Abeywardena's estimatior of repeatibility. Journal of Genetic, 16:247-254.

Vasconcellos MEC, Gonçalves PS, Paiva JR \& Valoris ACC (1985) Métodos de estimação do coeficiente de repetibilidade no melhoramento da seringueira. Pesquisa Agropecuária Brasileira, 20:433-437. 